\title{
Quasi-Non-Temporal Configuration Everettical Spaces
}

\section{Lebedev Yu.A.}

Bauman Moscow State Technical University, Moscow, Russia;

E-mail: Lebedev <lebedev@bmstu.ru>;

To formalize the philosophical axioms of everettics proposed the construction of quasi-non- temporal configuration everettical spaces, including both physical and psychoid aspects of the description of reality. Given adopted in everettics axiom of fractal similarity systems and subsystems relative state psychoid components of the proposed mathematical constructs formally symmetric physical components in their mathematical properties.

Keywords: configuration space, MW-interpretation of quantum mechanics, everettics, principle Amakko, timelessness Barbour, everettian, everettical world, psychoid essence, sklejka (fouzia), the Dirichlet function, Mathematical Universe of Tegmark.

DOI: $10.18698 / 2309-7604-2015-1-289-298$

\section{Introduction}

Currently, many-words interpretation of quantum mechanics [1] is becoming increasingly popular as the basis of the emerging worldview of the paradigm of the "Everett's many-words". Basic concepts everettics considered in the monograph "Many-Sided Universe" (the specific relation between everettics and mathematics - in a special Chapter of the third part of this monograph - "Everettical Pragmatics" [2, p. 484 - 541]). It is clear that further progress in understanding the Everett's many-words impossible without the use of mathematics as "traditional" and "designed" to describe the specific nature of this existential constructions.

Under "traditional use of mathematics" in the physical constructs refers to the methodology of extracting the physical meaning of the mathematical model of the phenomena. So understand the "correct" methodology of modern science Vladimir Kassandrov: "Indeed, the criterion of truth of our understanding of Nature can serve, perhaps, only our ability to understand and explain the structure of the universal numeric patterns discoverable by experience and independent of any subjective factors: certain interpretations of the results of experiments, systems, units, or theoretical assumptions" [3]. Indeed, those are the aspirations (unfortunately, rarely successful) and the common practice of "traditional" quantum mechanics.

Opposite the methodology involves the introduction of physically meaningful concepts and relations in a formal mathematical structure. 
This behavior reveals Archpriest Kirill Kopeikin: "the Convenience of mathematical language is in its formality: the same equation can describe many different phenomena. However, the flip side of this "formal convenience" is that a formal theory is open to its meaning" [4].

Often these trends coexist in the struggle with each other for the right to represent the "true" epistemology. From the point of view Everett tolerance, each of them is only one of the linear approximations of complex superposition of ways of knowing, including the "mixed" approaches. Last yet is only a formal expression of the principle of Amakko [5], but in everettics this approach promises to be particularly fruitful, as the Everett's many-words enters into consideration not only physically, but also logically incompatible existential construction.

\section{Everettical spaces}

\subsection{Geometrized Everettical spaces}

Consider possible variants of geometrical representations everettical spaces - sets of mappings relative state of holistic univers. The quality of the integrity of the univers allows not to consider physically devastating consequences of decoherence at the everett's branch. As noted by M. B. Mensky, "decoherence in this case does not occur, because the quantum world as a whole has no environment that could cause decoherence" [6, p. 16].

\subsubsection{Formalization of the concept of "everettical world"}

\section{Small everettians}

Was previously one of the proposed designs everettical spaces, the emphasis in the construction of which was made on account of the evolution of event-driven characteristics of Being $[7,8]$. In these works, the design of spaces that are metaspaces (Gödel's sense) in relation to the space-time of Minkowski.

Since time category refers to the quantum mechanical characterization of the relative state, which enables an individual to any process physical and psychoid components, it is advisable to consider formally atemporal mathematical construct relative state, containing the time in the implicit form as a parameter. This will allow further consideration of evolution in different temporal reference systems.

If you agree with the statement about Shakespeare that "All the world's a stage" [9]), we should expect that scene to everettic existence must have a very fancy design.

First of all, we introduce the class of "dual entity" - small everettian $e_{v_{i+j}}$ : 


$$
\psi_{q_{i}}+\Omega_{p_{j}}=e_{V_{i+j}}
$$

here:

$\Psi_{q_{i}}-$ quantum wave function

$\Omega_{p_{j}}$ - psychoid wave function ( $\Psi$ analogue psychoid in reality)

$\mathrm{q}, \mathrm{p}, \mathrm{i}, \mathrm{j}$ - identifiers (natural numbers).

The value of $e_{v_{i+j}}$ is called everettian, because it includes both poles everettical interpretation of the relative state of a holistic universe - physical $\left(\Psi_{q_{i}}\right)$ and psychoid $\left(\Omega_{p_{j}}\right)$ and is the representation of "everettical world" or, equivalently, the branches "altervers in Mensky". The reasons for this everettian named "small", will be clear from further.

The very nature of small everettian quaiatemporal because of its structural elements $\Psi_{q_{i}}$ and $\Omega_{p_{j}}$, describes the parameters of amplitude and phase, which have a physical meaning only together with the notion of a locally Newtonian time.

You should say why q and $\mathrm{p}$ be natural numbers. This design assumes that the set of states as the quantum and psychoid realities of the counting, and the set of many small everettian discretely. Indexing and quantum psychoid wave functions $\mathrm{i}$ and $\mathrm{j}$ individualizes them in accordance with the hypothesis of the existence of memory. The concept of the memory of the wave function, introduced by Everett [10, p. 458], individualizes as quantum and psychoid status and allows you to select how the physical objects in the quantum reality, and psychoid entities in reality psychoid.

This corresponds to the "ideology of the quantum universe" and is an evolution of the concept of the Barbour's universe as "Pinakothek states" - a chaotic meeting of the eternal and immutable "frames", which show all possible in this branch of the multiverse state of all its elements. [11] A separate issue is the method of ordering Barbour's Pinakothek - many small everettian.

The possible operations with small everettian allowed only the addition operation. The physical meaning of adding small everettian is the creation of their superposition.

As a result, for many, including "a" everettian, can be obtained the maximum superposition of the form:

$$
\sum_{i=1}^{i=a} \psi_{q_{i}}+\sum_{j=1}^{j=a} \Omega_{p_{j}}=e_{V_{a_{j}+a_{j}}}
$$


In addition, the summation in (2) can be carried out on small parts everettian from the set containing "a" elements by sets of (a-1), (a-2),.., 2 elements, and the set of summands in each case may be different. Thus, the set of sums of the form (2) will give a new set everettian $e_{v_{a_{i}+a_{j}}}$.

Everettian as complex numbers cannot be ordered according to the criterion of "more less". The only ordering criteria that have a clear physical meaning, is the criterion of "earlier later".

It is in the process of ordering, according to Barbour, consciousness and generates the time. With Everett the point of view of the active element in the creation of any reality is psychoid of "observer", so ways to organize, and, consequently, "times" should be very much. We can assume that at the lowest level of psychoid (the level of "inert matter"), with the creation of the classic realities of the physical world (CRPW) separating the quantum alternative consciousness (in the sense usually used by M. B. Mensky [6]), distinguishes between things at the level of small everettian (1). At a higher level (level of living) the difference is felt at the level of the extended set of small everettian (2). As these sensations are synchronized, creating a temporary order of any one variety (including "uniform mathematical time" Newton) - separate everettical issue. From a substantive point of view, a simple linear order can be interpreted both numerologically and abstract.

Geometrically the set of small everettian can be represented in the form of a set of points of the first quadrant of the Euclidean plane.

A separate issue is the mathematical essence points $\Psi_{q_{i}}$ and $\Omega_{p_{j}}$ on the coordinate axes of this plane. In the general case, every point on the coordinate axes is an individual or quantum or psychoid world, and "point" on the plane first quadrant - individual CRPW, "the frame of the Pinakothek of Barbour".

Since the specific structure of each everettical space depends on how the items are ordered $\Psi_{q_{i}}$ and $\Omega_{p_{j}}$, which, in turn, determined by the choice of the arrow of time for each index, $\mathrm{q}_{\mathrm{i}}$ and $\mathrm{p}_{\mathrm{j}}$, built space is quasiatemporary not only in terms of the nature of the wave functions, but in the sense of the order of the states of the memory settings for the constituent wave functions. This time is determined by how much everettical memory wave functions $\Psi_{q_{i}}$ and $\Omega_{p_{j}}$ and some additional rules build their vector arrow of time still to be determined.

As such the Euclidean plane there are no negative values, and there is no division because there is no "return value" (which corresponds to the degree minus one). 
It does not contain zero. "Nature abhors a vacuum" - zero small everettian corresponds to "absolute nothingness" CRPW. Geometrically axis $\Psi_{q_{i}}$ and $\Omega_{p_{j}}$ intersect at the point $\{1 ; 1\}$.

In a constructed mathematical model of the four structural elements of a Cartesian cut the Euclidean plane, only one identified as a tool to describe the everettical many-worlds . The plane is in general conformity with the model of "internal" and "external" observers (observers "first person" and "third person" in the terminology of James Hartle and Thomas Hertog [12]).

This design specifies the conditions for the existence and observation of our univers by "third party" - an external observer, an essential role in quantum mechanics was discussed by Everett [10], but introduced in this context in [13] and, independently, in [14].

However, the system introduced postulates displays an external observer from being and quantum, and psychoid, and classical realities. He is three-quarters of the Euclidean plane everettical space, but not fixed in any point and does not interact with everettian. Emerging physicalistic analogy with dark energy may be informative in further development of the proposed geometrized model of everettical space.

Based on these axioms space is the simplest quasiatemporary everettical space.

\subsubsection{Everettical multiplication. Full everettian}

The introduction of a multiplication operation of everettian introduces nonlinearity description, entangling and generates new mathematical, physical and psychoid entity.

Accept that in operation everettical multiplying the first factor is active early, "source action" on the second factor. In this regard, everettical multiplication is noncommunicational and nonassociative. Distributivity is preserved and provides the appearance of new entities.

Indeed, in the simplest case, multiplying two small everettian $e_{v_{1+1}}$ and $e_{v_{2+2}}$ are:

$$
\left(\Psi_{q_{1}}+\Omega_{p_{1}}\right) \cdot\left(\Psi_{q_{2}}+\Omega_{p_{2}}\right)=\Psi_{q_{1}} \cdot \Psi_{q_{2}}+\Psi_{q_{1}} \cdot \Omega_{p_{2}}+\Omega_{p_{1}} \cdot \Psi_{q_{2}}+\Omega_{p_{1}} \cdot \Omega_{p_{2}}
$$

All the summands in the right part of (3) - entangled state of reality $1\left(e_{v_{1+1}}\right)$ and $2\left(e_{v_{2+2}}\right)$. In everettics these states are called sklejka (fouzia) $[15,16]$. Then:

a)

b)
$\Psi_{q_{1}} \cdot \Psi_{q_{2}}$ - material sklejka (penetration, fouzia): influence of physical entities of reality 1 for the physical nature of reality 2 .

$\Psi_{q_{1}} \cdot \Omega_{p_{2}}$ - physical and mental sklejka (penetration, fouzia): influence of physical reality entity on 1 for psychoid essence of reality 2 . 
c) $\quad \Omega_{p_{1}} \cdot \Psi_{q_{2}}-$ mental-physical sklejka (penetration, fouzia): impact psychoid entity from actually 1 on the physical nature of reality 2 .

d) $\quad \Omega_{p_{1}} \cdot \Omega_{p_{2}}$ - mental sklejka (penetration, fouzia): impact psychoid entity of reality 1 on psychoid essence of reality 2 .

An interesting special case of eq. (3) which is multiplied with the same everettian, i.e. considers the square everettian $\left(e_{v_{1+1}}\right)^{2}$.

In this case, the material sklejka a) reflects the process of self-action of physical objects (for example, the impact of the electric charge of the electron itself), physical and mental sklejka b) corresponds to the representation of the physical impact of the object on the observer (the measurement process), mental-physical sklejka c) is "mental" influence of the observer on the physical object (such as telekinesis), mental sklejka d) reflects the process of reflection in psychology.

Everettical multiplication in the general case creates resultings from the multiplication of an arbitrary number of factors of the form $\Psi_{q_{i}}$ and $\Omega_{p_{j}}$ arranged in random order. The superposition of the obtained distribution of works will also be existential. The interpretation of the semantic content of such resultings from the multiplication and their superposition amounts is task specific everettical research. In philosophical terms, the study of such objects is dedicated to the works of A. Kosterin [17].

For description and identification of these objects must be entered in a special configuration Hilbert space $H_{e_{v}}$, each axis of which represents a corresponding member of superposition, but without indexes $\mathrm{i}$ and $\mathrm{j}$, and the origin is the point $\{1 ; 1 ; 1 \ldots\}$.

The ordering of the members of the index must comply with the rules imposed to organize small everettian.

Each point that does not lie on the axes $\Psi_{q_{i}}$ and $\Omega_{p_{j}}$ in the space $H_{e_{v}}$, will be in full everettian $E_{v_{i+j}}$ of some physico-psychoid object - specific "everettical world" with different "content" physical reality and psychoid reality.

Fantastic variety full everettians lets hope that after breeding the anthropic principle among them can be isolated worlds that are physically very different from our univers, but suitable for the existence of the mind "our type". This possibility had been foreseen and expressed in artistic form P. Amnuel in the novel "Three-universe". [18] 


\section{2. Algebraic everettical space}

All options have been considered "the stage of world action" are Geometrized everettical space designs. But there are also fundamentally different, deeper towards geometry, algebraic versions! And under their consideration, for example, by group V. Kassandrov already constructed an algebraic model worlds the Wheeler-Feynman, in which all the electrons and positrons univers are manifestations of a single particle [19] and the model of interaction of this particle with the observer [20].

Algebraic and approach is V. L. Janchilin to the description of motion [21]. Quantum motion in this approach is defined as a movement, discontinuous at every point of space. In this case, the nonlocality of quantum states is described by the Dirichlet function:

$\mathrm{X}(\mathrm{t})=\mathrm{X}_{1}$, if $\mathrm{t}$ is a rational number

$\mathrm{X}(\mathrm{t})=\mathrm{X}_{0}$, if $\mathrm{t}$ is an irrational number

This approach to the relationship of space (x) and Newtonian time (t) is essentially atemporal notion, because the t parameter is not dynamically (at time of this review no duration), and thermodynamically. Here, the time - marker of status and not of the process.

In this case, the Dirichlet function can easily be generalized so as to make an unambiguous and multivalued, even infinite-valued. For example, the function becomes ten, if you require to make it equal to some $\mathrm{M}$ the number after the decimal point in the decimal representation of a point $\mathrm{t}$ on the real axis, if this point is rational, and $\mathrm{N}$ number, if it is irrational [22].

The problem of using Newtonian (continuous) time when describing Everett's branching processes (discrete processes) is to establish the mechanism of ordering countable set of events on a continuous timeline.

Of course, these examples do not exhaust the geometric and algebraic possibilities of building a "Shakespearean scenes" to describe the theater of Existence. The philosophical question is - are they all suitable for the production of any "reality"? In other words, can the Mind to "think" something lacking a physical being? Or is all this and " external-Internal state", and realitys with numerical axes of magnitude "less than zero" and "greater than infinity", and the mathematics of psychoid in which "the sum of suffering gives the absurd" [23, p. 113] are only "mind game"? But, say N. Bourbaki attributed to Hermite following sentence: "the Number, functions and other mathematical concepts like animals in a zoo: they can admire, but cannot be changed: they ARE!". Today M. Tegmark suspected Hermite's animals not only in existential, but in physical reality level, and shrewdly took them to many-worlds fourth, the mathematical level of everettical manyworlds [24]. 


\section{Conclusion}

Presents design everettical spaces are not calculated mathematical models because include meaningful nominees (in particular related to psychoid described structures), which currently do not have a quantitative description. Furthermore, the analysis of the introduced axioms have shown that the proposed configuration space to contain the area, a meaningful interpretation of the States in which it is impossible neither in physics nor in the philosophy and refers to the fourth type of many-worlds by M. Tegmark - mathematical many-worlds.

A pragmatic sense of this kind of modeling and describing the Everett's many-worlds is that opens up a new directory of your consideration of Entity and encourages their inclusion in a holistic view of the universe.

\section{Thanks}

The author honors the memory of the recently deceased M. B. Mensky, mental dialogue which significantly affected the progress and outcome of work and also grateful to V. V. Kassandrov and M. H. Shulman for a discussion of some conceptual issues relevant to it.

\section{References}

1. Vaidman L. (2002). Many-Worlds Interpretation of Quantum Mechanics. Stanford Encyclopedia of Philosophy. Retrieved from: http://plato.stanford.edu/entries/qmmanyworlds/

2. Lebedev Y.A. (2010). Mnogolikoe mirozdanie. Everetticheskaja pragmatika [The Everettical Pragmatics]. Moscow: LeGe Publ.

3. Kassandrov V.V. (2012). Chislo-struktura-materija: na puti k radikal'noj pifagorejskoj metodologii fundamental'nogo estestvoznanija. [Number-structure-matter: towards a radical Pythagorean methodology of fundamental natural science]. Metafizika [Metaphysics], N 1(3), $85-102$.

4. Kopejkin K. (2012). Harmonia Mundi: ot Pifagora do Pauli [Harmonia Mundi: From Pythagoras to Pauli]. Metafizika [Metaphysics], N 1(3), 39 - 59.

5. Lebedev Y.A. (2012). Princip Amakko [The principle of Amacco]. Retrieved from: http://milkywaycenter.com/everettica/Leb021212.pdf

6. Mensky M.B. (2011). Soznanie I kvantovaja mehanika. [Consciousness and quantum mechanics]. Frjazino [Fryazino]: Vek-2 Publ. 
7. Lebedev Y.A., Amnuel P.R., Dulphan A.Ya. (2015). «Infinite-Dimensional Multievents Space-Time of Minkowski and Everett's Axiom of Parallelism». American Journal of Modern Physics. Special Issue: Physics of Time: Theory and Experiment, Vol. 4, No. 2-1, 1-8.

8. Lebedev Y.A. (2014). Model' beskonechnomernogo mul'tisobytijnogo prostranstva-vremeni Minkovskogo ifizicheskij smysl everetticheskih vetvlenij I skleek. [Multievent model infinitedimensional space-time of Minkowski and Everett physical meaning of the branching and fuzions]. Matematicheskie struktury I modelirovanie [Mathematical structures and modeling], $\mathrm{N} 4(32), 13-22$.

9. Shakespeare W. «As You Like It», spoken by Jaques in Act II Scene VII.

10. Everett H. (1957). "Relative State" Formulation of Quantum Mechanics. Reviews of Modern Physics, Vol 29, 454-462.

11. Barbour J. (1999). The end of time: the next revolution in our understanding of the universe. London : Weidenfeld \& Nicolson.

12. Hartle J., Hertog T. (2015). The Observer Strikes Back. arXiv, 1503.07205v1 [gr-qc].

13. Hawking S.W., Hertog T. (2006). Populating the Landscape: A Top Down Approach. Phys. Rev., D, 73, 123527.

14. Lebedev Y.A. (2007). Nelinejnye semanticheskie aspekty kvantovomehanicheskoj koncepcii X.Everetta I perspektivy razvitija everettiki. [Nonlinear semantic aspects of the concept of relative state $\mathrm{H}$. Everett and prospects of development of everettica]. Matematicheskie struktury I modelirovanie [Mathematical structures and modeling], N 17, 53-71.

15. Lebedev Y.A. (2000). Neodnoznachnoe mirozdanie. [Ambivalent Universe]. Kostroma: Infopress Publ.

16. Lebedev Y.A., Amnuel P.R., Dulfan A.Ya. (2013). The Everett axiom of parallelism. arXiv, 1304.0310v1

17. Kosterin A. (2015). O vozmozhnosti vlijanija al'terversov. [On the possibility of mutual influence of altivers]. Retrieved from: http://milkywaycenter.com/everettica/AK120315D.pdf.

18. Amnuel' P. (2004). Trivselennaja. [Three universe]. Novomoskovsk.

19. Kassandrov V.V., Khasanov I.S., Markova N.V. (2014). Algebraic dynamics on a single worldline: Vieta formulas and conservation laws. arXiv, 1402.6158v2 [math-ph]

20. Kassandrov V.V., Khasanov I.S., Markova N.V. (2014). Collective Lorentz invariant dynamics on a single "polynomial" worldline. arXiv, 1501.01606v1 [physics.gen-ph] 
21. Janchilin V.L. (2010). Kvantovaja nelokal'nost'. [Quantum nonlocality]. Moscow: URSS Publ.

22. Lebedev Y.A. (2015). "Levenguki" i "N'jutony" kvantovogo mira. ["Levenhuk" and "Newtons" of the quantum world]. Mlechnyj put'. [Milky way], N1(12), 218 -240.

23. Brodsky I.A. (1994). Izbrannye stihotvorenija. [Selected Poems]. Moscow: Panorama Publ.

24. Tegmark M. (2007). The Mathematical Universe. Foundations of Physics, Vol.38 (2), 101150. 